\title{
An androgen receptor negatively induced long non-coding RNA ARNILA binding to miR-204 promotes the invasion and metastasis of triple-negative breast cancer
}

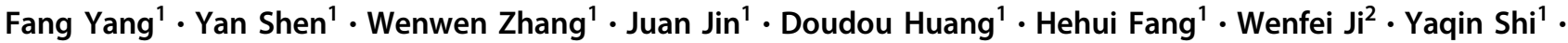 \\ Lin Tang ${ }^{1} \cdot$ Weiwei Chen ${ }^{1} \cdot$ Guohua Zhou ${ }^{3} \cdot$ Xiaoxiang Guan ${ }^{1,2,4}$
}

Received: 24 August 2017 / Revised: 9 April 2018 / Accepted: 18 April 2018 / Published online: 29 May 2018

(c) ADMC Associazione Differenziamento e Morte Cellulare 2018

\begin{abstract}
Androgen receptor (AR) is emerging as a novel prognostic biomarker in triple-negative breast cancer (TNBC), but the underlying mechanisms remain unknown. As accumulating evidence has shown that long non-coding RNAs (lncRNAs) regulate important cancer hallmarks, we hypothesised that AR-regulated lncRNAs might play roles in TNBC progression. Here, we performed experiments with or without DHT treatment in three TNBC cell lines, and we identified an AR negatively induced lncRNA (ARNILA), which correlated with poor progression-free survival (PFS) in TNBC patients and promoted epithelial-mesenchymal transition (EMT), invasion and metastasis in vitro and in vivo. Subsequently, we demonstrated that ARNILA functioned as a competing endogenous RNA (ceRNA) for miR-204 to facilitate expression of its target gene Sox4, which is known to induce EMT and contribute to breast cancer progression, thereby promoting EMT, invasion and metastasis of TNBC. Our findings not only provide new insights into the mechanisms of lncRNA in regulating AR but also suggest ARNILA as an alternative therapeutic target to suppress metastasis of TNBC patients.
\end{abstract}

\section{Introduction}

Triple-negative breast cancer (TNBC), characterised by a lack of the oestrogen receptor (ER), progesterone receptor (PR) expression and human epidermal growth factor receptor 2 (HER2) amplification, is the most aggressive subtype of tumours associated with poor clinical outcomes

Edited by RA Knight.

Electronic supplementary material The online version of this article (https://doi.org/10.1038/s41418-018-0123-6) contains supplementary material, which is available to authorized users.

Xiaoxiang Guan

xguan@nju.edu.cn

1 Department of Medical Oncology, Jinling Hospital, Medical School of Nanjing University, Nanjing, China

2 Department of Medical Oncology, Jinling Clinical College of Nanjing Medical University, Nanjing, China

3 Department of Pharmacology, Jinling Hospital, Medical School of Nanjing University, Nanjing, China

4 Department of Oncology, The First Affiliated Hospital of Nanjing Medical University, Nanjing 210029, China
$[1,2]$. Few effective prognostic markers and targeted approaches are currently available for TNBC. Recently, the oral poly (adenosine diphosphate-ribose) polymerase (PARP) inhibitor olaparib has shown promising antitumour activity in HER2-negative patients with a germline BRCA mutation [3]. BRCA germline mutations have a high prevalence in the TNBC subtype, and thus, olaparib represents the first targeted therapy in this type of cancer. There is an urgent need to develop more therapeutic targets and novel biomarkers to treat TNBC. Androgen receptor (AR), a nuclear receptor showing high structural, functional and topographic similarity to ER and PR, is commonly expressed in breast cancer and has increasingly been shown to be involved in tumour progression, recurrence and metabolic reprogramming [4]. High expression of AR was reported to correlate with prolonged survival of TNBC [5-7]. Nevertheless, the underlying mechanisms of AR in regulating tumour progression remain elusive.

Long non-coding RNAs (lncRNAs) are defined as the non-protein-coding transcripts greater than $200 \mathrm{nt}$ in length [8]. LncRNAs were shown to have broad functional roles in regulating important cancer processes, including proliferation [9], apoptosis [10], metastasis [11], and drug resistance [12]. A wide range of models have been proposed to explain 
Fig. 1 AR expression in TNBC and association with survival. a Correlation between AR expression levels and PFS (logrank test) in 88 TNBC patients. b Correlation between AR expression levels and RFS (logrank test) in basal-like breast cancer from Kaplan-Meier Plotter (http://www.kmplot. com/analysis/). Patients were stratified into low and high AR expression based on an auto select best cutoff. c Forest plots of hazard ratios for PFS of ARpositive $v s$ AR-negative groups. d AR mRNA levels from TCGA database, including 14 TNBC tumour and paired normal tissues
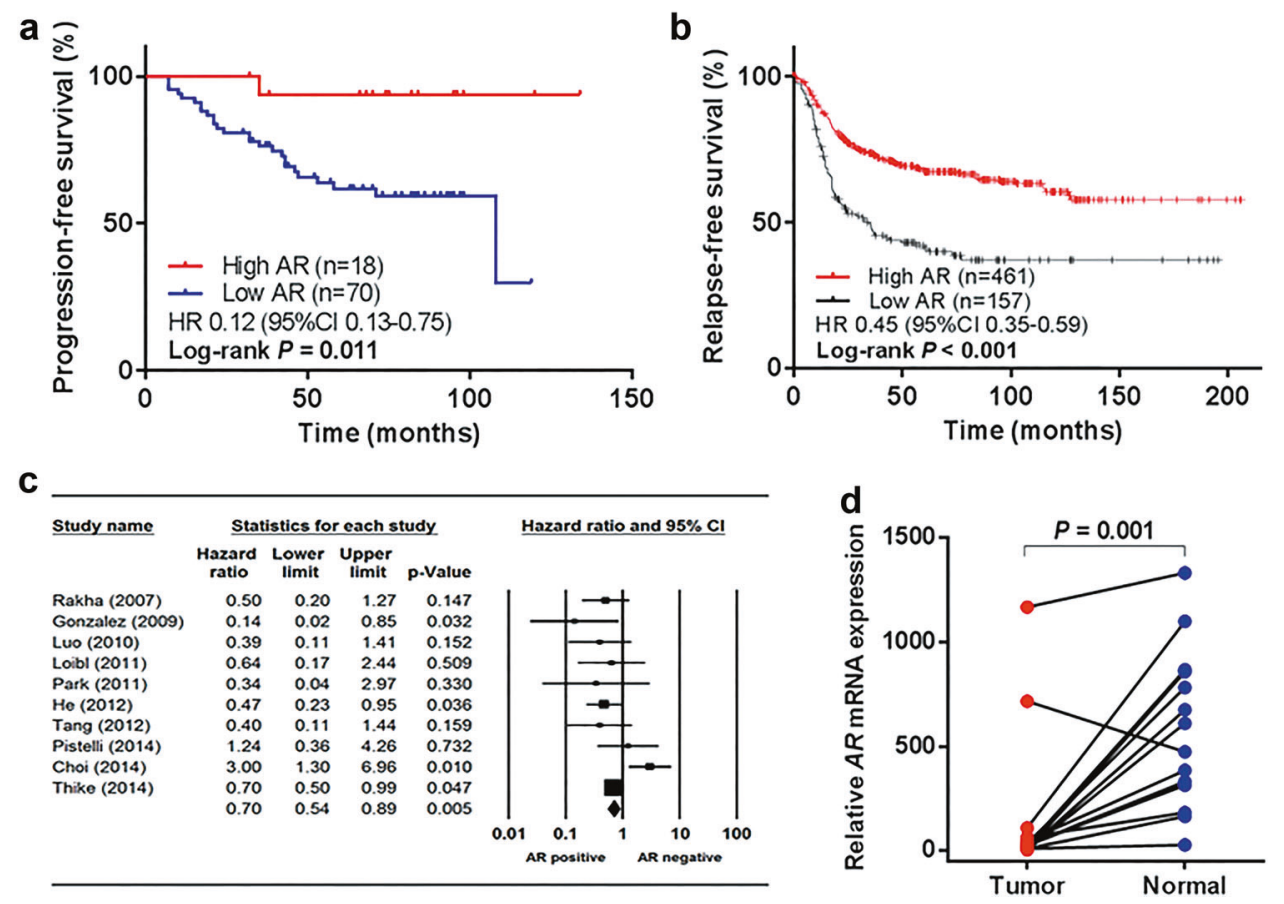

the precise mechanisms by which lncRNAs function [13]. These molecules can regulate transcription by serving as guides that recruit transcriptional co-regulators or chromatin-modifying enzymes to a specific DNA region [10] or form ribonucleoprotein complexes by acting as molecular scaffolds to bridge together regulatory proteins [14]. Additionally, lncRNAs may act as decoys that bind microRNAs (miRNAs) or proteins to control the functions of regulatory molecules [15]. Collectively, lncRNAs can function as tumour suppressors or oncogenes by altering these functions to promote or suppress tumour formation, progression and metastasis.

In this study, we aimed to identify the AR-related IncRNAs and investigated the contributions of these lncRNAs to invasion and epithelial-mesenchymal transition (EMT) of TNBC cells and their roles in TNBC metastasis. Here, we identified an AR negatively induced lncRNA (ARNILA) (Ensembl: ENST00000431557) in TNBC and found it was correlated with poor progressionfree survival (PFS). Further studies revealed its critical roles in invasion, EMT and metastasis. EMT is the first crucial step in metastasis formation; this process facilitates cancer cell motility and dissemination and promotes dedifferentiation, growth arrest, migration and invasion [16]. We found the ARNILA could sequester miR-204 by functioning as a competing endogenous RNA (ceRNA) to upregulate the miR-204-targeted gene Sox4. Sox4 was recently shown to be abnormally overexpressed in TNBC and correlated with this subtype. Additionally, this gene is considered to play a crucial role in breast cancer progression by orchestrating EMT $[17,18]$. We demonstrated that Sox4 is responsible for ARNILA-mediated migration and EMT.

\section{Results}

\section{Positive expression of AR is correlated with better prognosis in TNBC}

Recently, preclinical and early clinical studies have shown that AR may be a prognostic marker in diagnostics and therapy. We first assessed the relationship between AR and PFS in TNBC in 88 TNBC samples. We found that higher expression of AR was significantly associated with a prolonged PFS (hazard ratio (HR), 0.12; 95\% confidence interval (CI), 0.13-0.75; $P=0.011$ ) (Fig. 1a). We confirmed that positive AR expression was correlated with a significant improvement in relapse-free survival (RFS) (HR, 0.45 ; 95\%CI, $0.35-0.59 ; P<0.001)$ in 618 TNBC samples with the online tool Kaplan-Meier Plotter (http://www. kmplot.com/analysis/) [19] (Fig. 1b). Moreover, our previous meta-analysis of nine retrospective studies that included 2463 TNBC patients revealed that higher AR expression was correlated with a prolonged PFS (HR, 0.70; 95\%CI, 0.54-0.89; $P=0.005$ ) [20] (Fig. 1c). Moreover, we extracted the expression data for AR from The Cancer Genome Atlas (TCGA) database, which indicated that tumour tissues exhibited significantly decreased AR expression compared with paired normal tissues in 14 TNBC patients $(P=0.001)$ (Fig. 1d). 

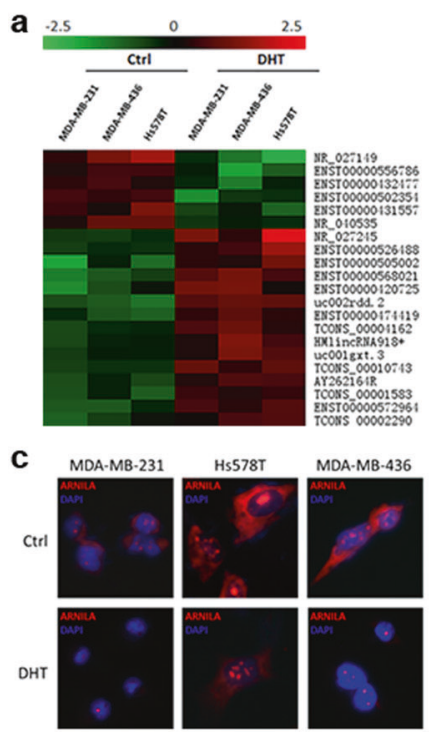

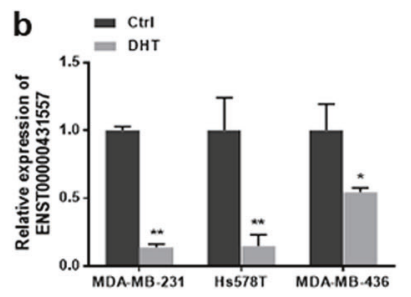

f
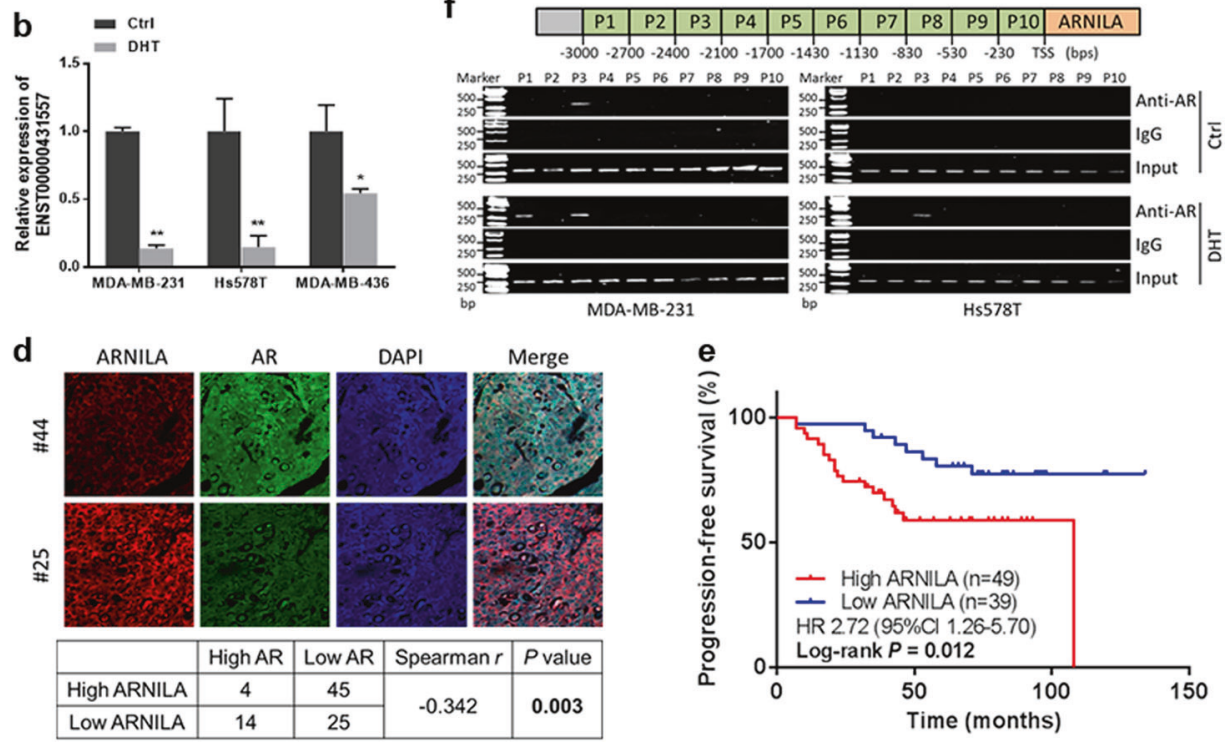

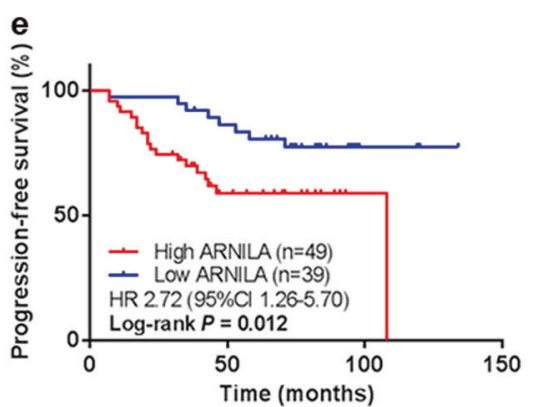

Fig. 2 Identification of the AR negatively induced lncRNA ARNILA. a Heatmap representation of IncRNA microarray data for MDA-MB231, Hs578T and MDA-MB-436 cells treated with or without DHT (fold change $\geq 2.0, P<0.05$ ). $\mathbf{b}$ Expression of ARNILA was assayed by qRT-PCR in MDA-MB-231, Hs578T and MDA-MB-436 cells treated with or without DHT. ARNILA levels were normalised to GAPDH. c FISH images showing cellular localisation of ARNILA in MDA-MB231 and Hs578T cells. d Upper: representative FISH images for ARNILA and AR in paraffin-embedded tissue of TNBC patients.

\section{Identification of AR negatively induced IncRNA ARNILA}

Although AR shows potential prognostic value in TNBC, the mechanisms for this correlation remain unknown. Considering the emerging roles of lncRNAs in EMT, invasion and metastasis of breast cancer [21-25], we hypothesised that AR-regulated IncRNAs play a role in TNBC progression. To identify AR-relevant lncRNAs in TNBC, we therefore determined the expression profiles of lncRNAs in three cell lines (MDA-MB-231, Hs578T, MDA-MB-436) treated with or without dihydrotestosterone (DHT). We identified 21 differentially expressed lncRNAs (15 upregulated and 6 downregulated) (fold change $\geq 2.0$, $P<0.05$ ) (Fig. 2a). Further qRT-PCR analysis confirmed the high-throughput profiling results. The lncRNA ENST00000431557 was significantly decreased in DHTtreated cells compared to control cells (Fig. 2b). Accordingly, we chose this uncharacterised lncRNA for further study and named it ARNILA (androgen receptor negatively induced lncRNA). Fluorescence in situ hybridisation (FISH) assays confirmed that ARNILA was primarily located in the cytoplasm of TNBC cells (Fig. 2c) and tissues (Fig. 2d, upper). Notably, cytoplasmic ARNILA expression was significantly altered by DHT, suggesting that ARNILA could act as a ceRNA to absorb miRNAs. A negative
Lower: the correlation of ARNILA and AR expression in the 88 TNBC patients. e Correlation between ARNILA expression levels and PFS (log-rank test) in 88 TNBC patients. f Upper: The primer pair locations within the ANRILA promoter. Lower: ChIP assay of the enrichment of AR on ARNILA promoter in MDA-MB-231 and Hs578T cells. Positive and negative controls are indicated as input and IgG, respectively. The results are presented as the mean $\pm \mathrm{SD}$. $* P<0.05, * * P<0.01$

correlation was found between AR and ARNILA expression in 88 TNBC cases $(P=0.003)$ (Fig. 2d). Furthermore, high ARNILA expression was correlated with poor PFS (HR, 2.72; 95\% CI, $1.26-5.70 ; P=0.012$ ) in TNBC (Fig. 2e).

It has been reported that AR could serve as a transcriptional repressor by binding to the promoters of target genes [26]. Increased binding of AR to the promoter of ARNILA was observed by chromatin immunoprecipitation (ChIP) assays (Fig. 2f). Two binding sites contain the sequence corresponding to the core motifs of the androgen response element, suggesting that AR may be a transcription factor that downregulates ARNILA. Rapid amplification of cDNA ends (RACE) assays showed that ARNILA is composed of two exons with a full length of 930nt located on chromosome 2 (Supplementary Figure S1).

\section{ARNILA functions as a ceRNA for miR-204 to facilitate Sox4 expression}

To test the hypothesis that ARNILA could act as a ceRNA to regulate cancer progression, we first predicted the potential binding sites for 2588 human miRNAs [27] on ARNILA by miRanda [28] and next identified the TNBC survival-associated miRNAs by Kaplan-Meier Plotter [29]. The bioinformatics analysis revealed the top ten 


\begin{tabular}{|c|c|c|}
\hline \multicolumn{1}{|c|}{ miRNA } & Max Score & OS \\
\hline hsa-miR-18a-3p/5p & 164.00 & Negative \\
\hline hsa-miR-18b-5p & 156.00 & Negative \\
\hline hsa-miR-204-3p/5p & 145.00 & Positive \\
\hline hsa-miR-33b-3p/5p & 144.00 & Negative \\
\hline hsa-miR-23b-5p & 143.00 & Positive \\
\hline hsa-miR-92b-3p & 143.00 & Positive \\
\hline hsa-miR-584-5p & 142.00 & Positive \\
\hline hsa-miR-423-5p & 140.00 & Negative \\
\hline hsa-miR-548q & 140.00 & Positive \\
\hline hsa-miR-148a-3p & 140.00 & Negative \\
\hline
\end{tabular}

b

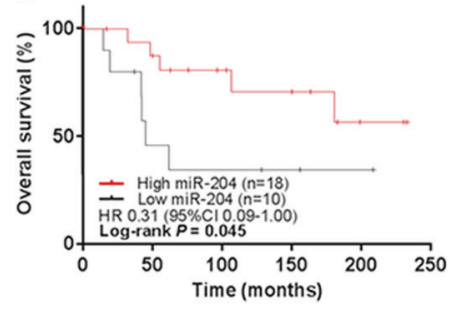

g
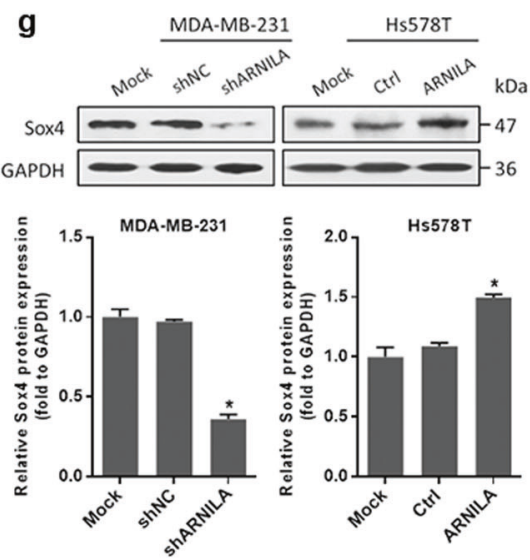

h
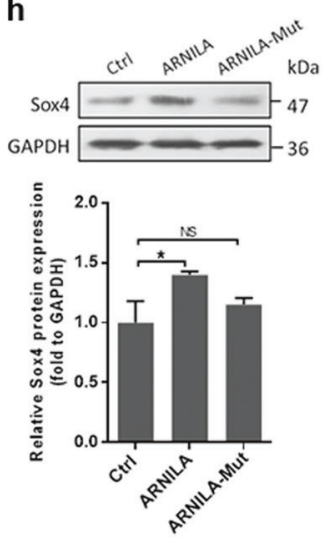
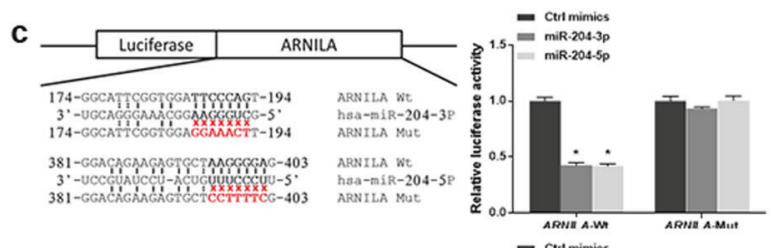

d
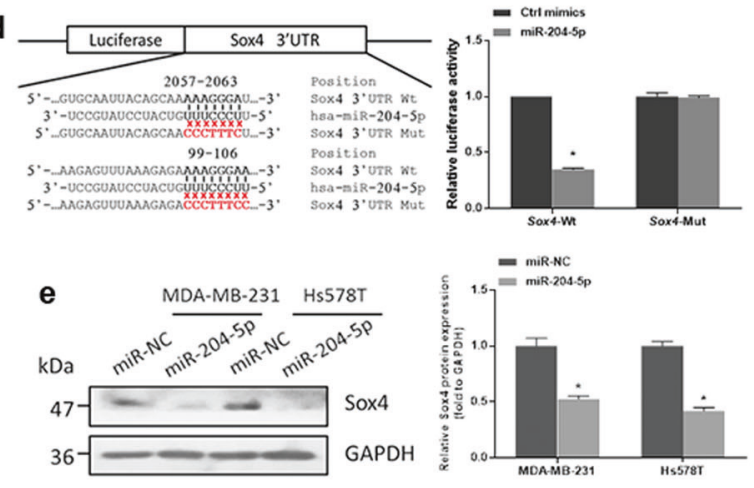

$\mathbf{f}$
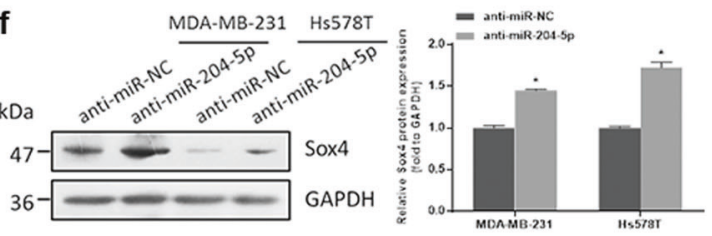

Fig. 3 ARNILA upregulates Sox4 via binding to miR-204. a The top ten putative miRNAs binding to ARNILA sorted according to the max score predicted by miRanda software. The association between miRNA expression and OS in untreated TNBC patients was derived from Kaplan-Meier Plotter (log-rank test). b Correlation between AR expression levels and OS (log-rank test) in TNBC patients from Kaplan-Meier Plotter. Patients were stratified into low and high AR expression based on auto select best cutoff. c Left: putative miR-204 MREs in the $3^{\prime}$ UTR of ARNILA generated by miRanda software. The seed sequences are shown in bold and mutant sequences shown in red. Right: luciferase activity of ARNILA-Wt and ARNILA-Mut upon transfection of the indicated miRNA mimics in 293T cells. Data are presented as the ratio of Renilla luciferase activity to firefly luciferase activity. d Left: putative miR-204 MREs in the 3'UTR of Sox 4 generated by the TargetScan database. The seed sequences are shown in bold and mutant sequences shown in red. Right: luciferase activity of Sox4-Wt and Sox4-Mut upon transfection of the indicated miRNA mimics in 293T cells. Data are presented as the ratio of Renilla luciferase activity to firefly luciferase activity. e, f Left: western blotting assay of Sox4 expression in MDA-MB-231 and Hs578T cells following miR-204 mimic (e) or inhibitor (f) transfection. Right: quantification of protein levels shown in the left panel. $\mathrm{g}$ Upper: western blotting assay of Sox 4 expression in MDA-MB-231 and Hs578T cells after transfection with the indicated plasmid. Lower: quantification of protein levels shown in the upper panel. h Upper: western blotting assay of expression of Sox 4 in Hs578T cells after transfection with the indicated plasmids. Lower: quantification of protein levels shown in the upper panel. The results are presented as the mean \pm SD. $* P<0.05$. NS indicates no significant difference

mimics $(P<0.05)$ (Fig. 3d). The luciferase reporter assays demonstrated direct interactions between miR-204 and ARNILA, and miR-204 and Sox4. Notably, the same binding sites shared by ARNILA and Sox 4 on miR-204 indicate that ARNILA functions as a ceRNA for miR-204 to facilitate Sox 4 expression. Furthermore, we found that miR204 inhibitor strongly increased ARNILA levels compared with miR-NC, as shown by qRT-PCR (Supplementary Figure S2a). A significant reduction of Sox 4 protein in cells transfected with miR-204 mimics was demonstrated by western blot assays (Fig. 3e). Conversely, miR-204 inhibitor transfection resulted in a strong increase in Sox4 protein level (Fig. 3f). ARNILA knockdown decreased Sox4 protein expression, while ARNILA overexpression increased Sox 4 protein expression (Supplementary Figure S2b-c, Fig. 3g). Notably, mutations in the miR-204-binding site on ARNILA abolished its regulatory effect on Sox4 

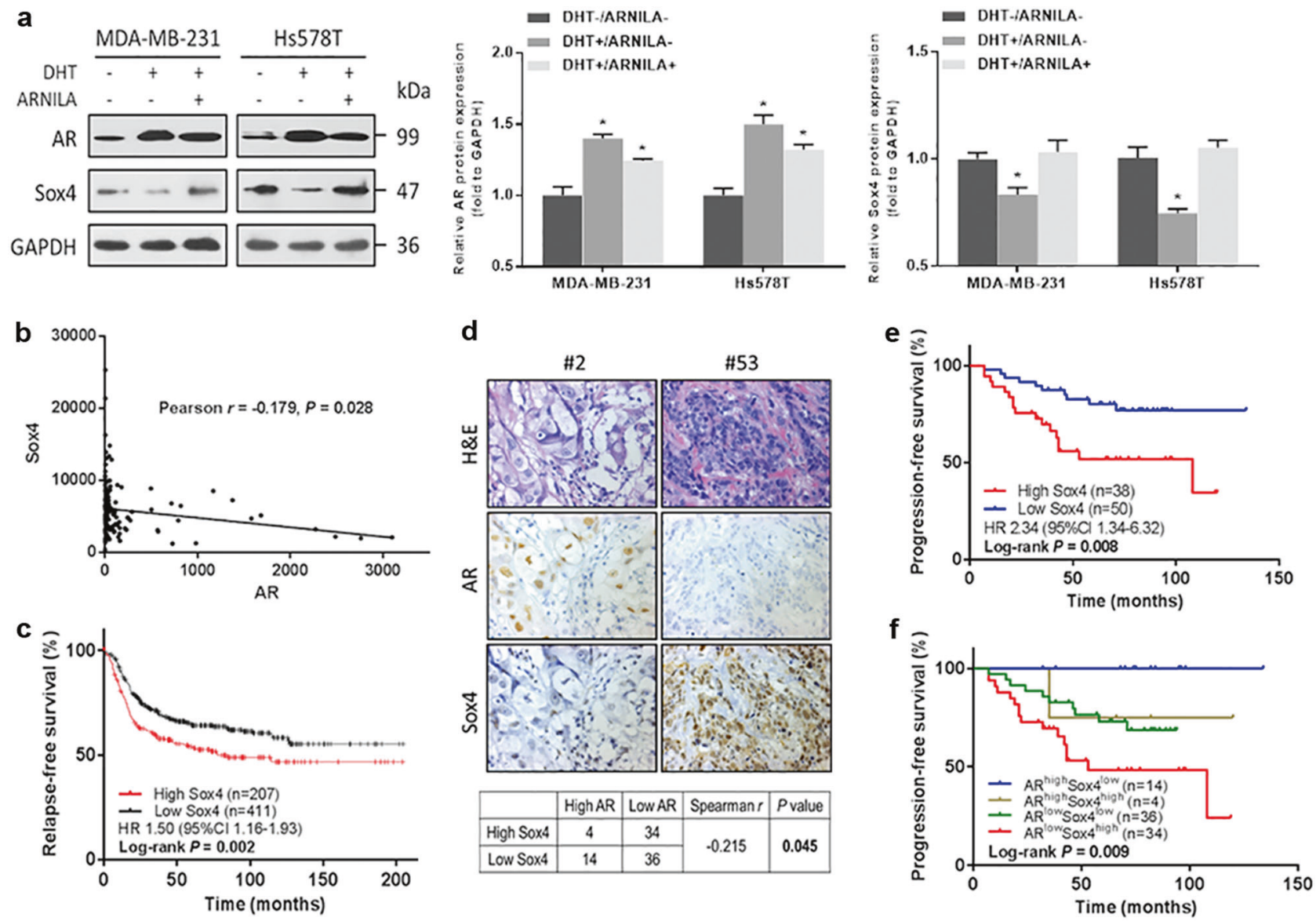

Fig. 4 Sox 4 is inversely correlated with AR and better survival. a Left: western blotting assay of expression of AR and Sox4 in MDA-MB231 and Hs578T cells following the indicated treatment. Middle and right: quantification of protein levels shown in the left panel. The results are presented as the mean $\pm \mathrm{SD}$. $* P<0.05$. b AR and Sox 4 expression from TCGA database of 151 TNBC patients. c Correlation between Sox4 expression levels and RFS (log-rank test) in TNBC patients from Kaplan-Meier Plotter. Patients were stratified into low

and high Sox4 expression based on auto select best cutoff. d Upper: representative IHC images for AR and Sox4 in paraffin-embedded tissue of TNBC patients. Lower: the correlation of AR and Sox4 expression in the 88 TNBC patients. e Correlation between Sox 4 levels and PFS (log-rank test) in 88 TNBC patients. f Correlation between AR-Sox 4 combined levels and PFS (log-rank test) in 88 TNBC patients

(Fig. 3h). Given the regulatory efficiency of ARNILA in Sox4, we hypothesised that ARNILA could also affect other miR-204 targets. Similarly, ARNILA knockdown decreased additional four miR-204 target proteins (BCL2, RAB22A, SIRT1, and FOXA1), while ARNILA overexpression increased the expression of these proteins (Supplementary Figure S3). Although RUNX2 was also shown to be a validated targeted gene of miR-204, its expression was not altered by ARNILA knockdown or overexpression (Supplementary Figure S3). We hypothesised that ectopic expression or knockdown of ARNILA exerted a limited change of miR-204, which is insufficient to repress all target genes at the same time. Additionally, RUNX2 expression could be controlled by other regulatory mechanisms that overwhelmed the effect of the miR-204 alteration. Collectively, these data indicate that ARNILA might function as a molecular sponge of miR-204 to upregulate Sox4 expression.

\section{Sox4 is inversely correlated with AR and predicts poor clinical outcome in TNBC}

The lncRNA ARNILA was downregulated by AR, indicating that AR is upstream of Sox 4 in the pathway. DHT significantly suppressed Sox 4 protein level by activating AR in TNBC cells, whereas ARNILA overexpression abolished this suppressive effect (Fig. 4a), indicating that AR negatively regulates Sox 4 by inhibiting ARNILA. We analysed RNA-seq data of TCGA database by comparing the AR and Sox 4 mRNA levels and found that Sox 4 was inversely correlated with AR in 151 TNBC patients $(P=$ 0.028) (Fig. 4b). Moreover, the patients with high Sox4 expression showed a significantly reduced RFS compared with that of patients with low Sox 4 expression in TNBC by Kaplan-Meier Plotter [29] (HR, 1.50; 95\%CI, 1.16-1.93; $P=0.002$ ) (Fig. 4c). To further examine the correlation between AR and Sox4, we analysed the protein expression 
a
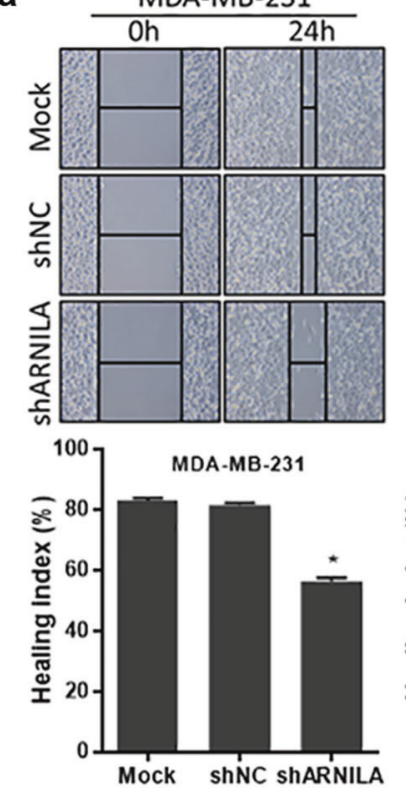
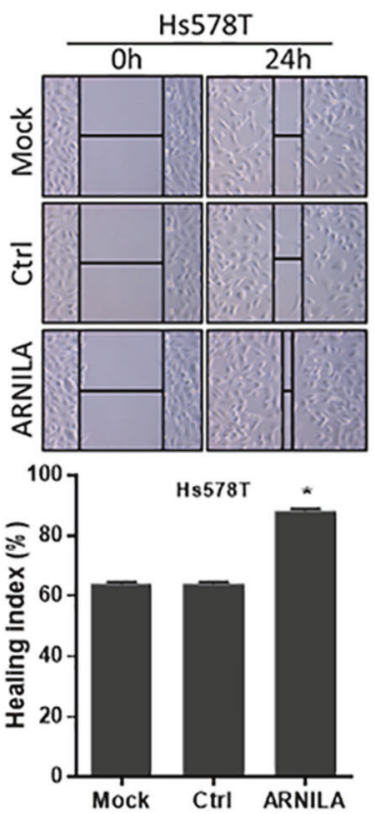
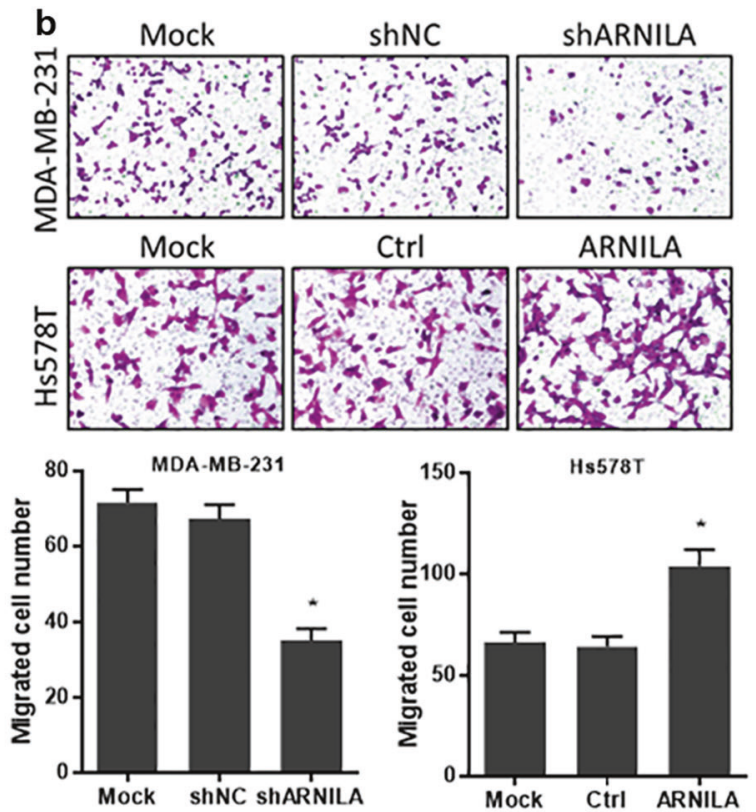

C
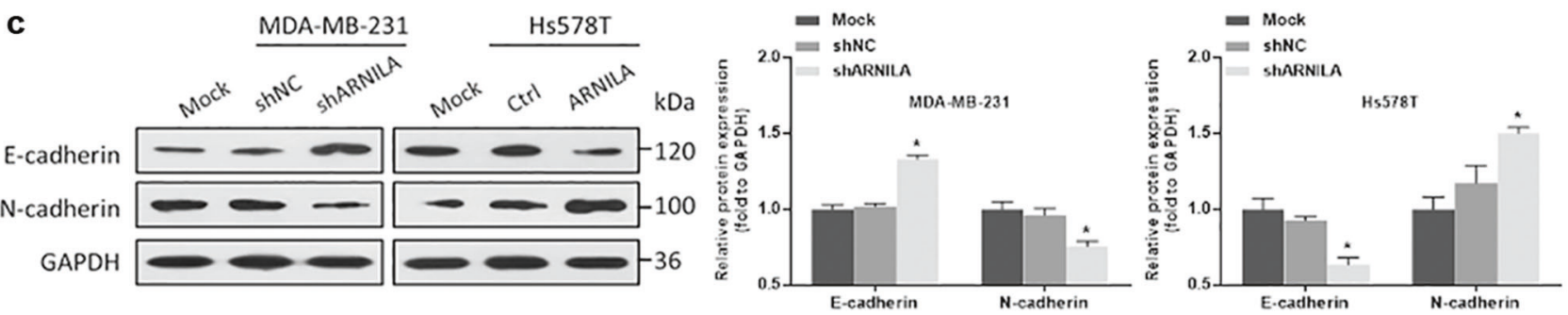

Fig. 5 ARNILA promotes migration, invasion and EMT in vitro. a Upper: wound healing assays of MDA-MB-231 and Hs578T cells following the indicated transfections. Lower: quantification of wound closure for cells in the upper panel. b Upper: transwell assays of MDA-MB-231 and Hs578T cells following the indicated transfections.

in $88 \mathrm{TNBC}$ tissue samples (Fig. 4d). Sox4 expression was negatively correlated with $\mathrm{AR}$ expression $(P=0.045)$ (Fig. 4d). Furthermore, Sox 4 was correlated with poor PFS (HR, 2.34; 95\%CI, 1.34_6.32; $P=0.008$ ) (Fig. 4e). Patients with different Sox 4 and AR levels showed significant differences in PFS $(P=0.009)$, and notably, patients with high Sox4 and low AR exhibited a substantially shorter PFS than those with low Sox4 and high AR (HR, 0.00; 95\%CI, 0.07 $-0.57 ; P=0.003$ ) (Fig. 4f). Taken together, these data serve as additional evidence confirming the regulation of Sox4 by ARNILA. Moreover, the findings indicate that AR has an impact on TNBC clinical outcome by negatively regulating Sox4.

\section{ARNILA promotes migration, invasion and EMT in vitro}

Sox4 has been shown to induce the EMT programme [17, 18], and therefore, we hypothesised that ARNILA might
Lower: quantification of migration by cells in the lower panel. c Left: western blotting assay of expression of E-cadherin and N-cadherin in MDA-MB-231 and Hs578T cells following the indicated treatments. Middle and right: quantification of protein levels shown in the left panel. The results are presented as the mean \pm SD. $* P<0.05$

also play a role in EMT. We knocked down ARNILA in MDA-MB-231 cells and overexpressed it in Hs578T cells to further investigate the functions of ARNILA in TNBC progression. ARNILA knockdown significantly decreased the migration of MDA-MB-231 cells, and ARNILA overexpression accelerated the speed of wound closure in Hs578T cells (Fig. 5a). Moreover, silencing ARNILA inhibited invasion, while ectopic expression of ARNILA significantly enhanced invasion as shown by transwell invasion assays (Fig. 5b). MDA-MB-231 cells transfected with a shARNILA plasmid showed significantly increased expression of the epithelial marker E-cadherin, along with a prominent decrease in the mesenchymal marker $\mathrm{N}$-cadherin. Conversely, the gain-of-function study in Hs578T cells revealed that ARNILA overexpression reduced the epithelial marker E-cadherin, along with increased expression of the mesenchymal marker N-cadherin (Fig. 5c). In summary, these data suggest that ARNILA is a positive regulator of migration, invasion and EMT in TNBC cells. 
a
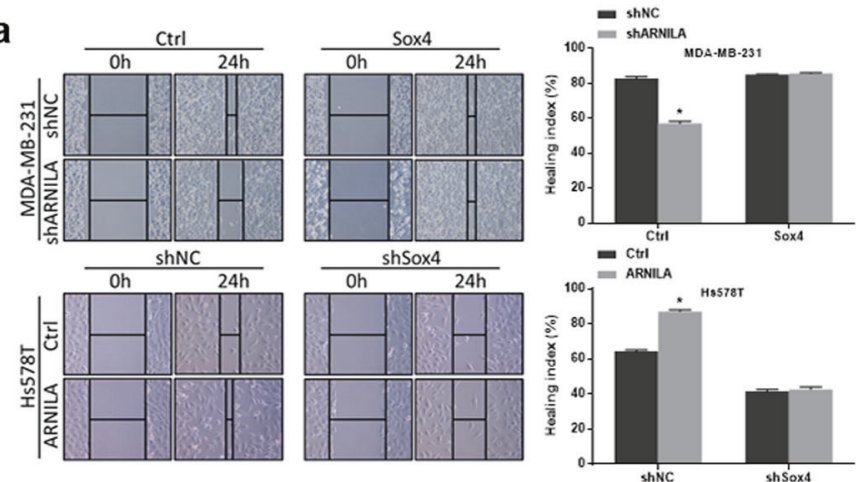
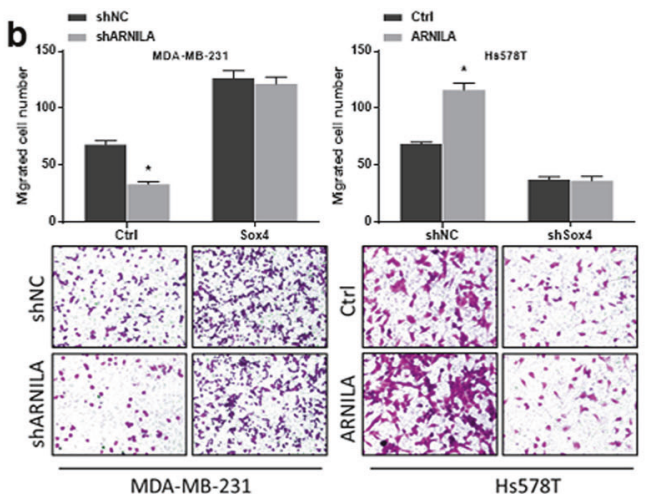

C

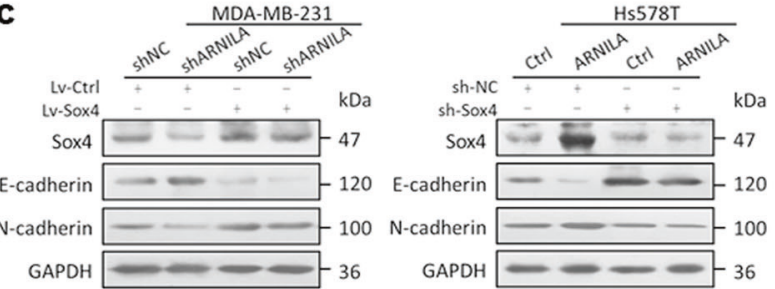

Fig. 6 Sox 4 is responsible for ARNILA-mediated migration, invasion and EMT in vitro. a Left: wound healing assays of MDA-MB-231 and Hs578T cells following the indicated transfections. Right: quantification of wound closure for cells in the left panel. b Lower: transwell assays of MDA-MB-231 and Hs578T cells following the indicated transfections. Upper: quantification of migration by cells in the lower

\section{Sox4 is responsible for ARNILA-mediated migration, invasion and EMT in vitro}

We further investigated the mechanisms by which ARNILA promoted malignant processes. Given that ARNILA functions as a ceRNA to upregulate Sox 4 expression, we performed functional rescue experiments to determine whether ARNILA promoted tumour progression in a Sox4dependent manner. Plasmids with shNC (short hairpin RNA of normal control) or shARNILA were transfected into MDA-MB-231 cells that stably ectopically expressed Sox4 or the control, whereas Hs578T cells that stably ectopically expressed ARNILA or the control were transfected with shNC or shSox 4 plasmids. Notably, restoration of Sox 4 rescued the wound healing rate and invasive capacity in ARNILA-knockdown MDA-MB-231 cells, while Sox4 knockdown significantly abolished ARNILA-mediated migration and invasion in Hs578T cells (Fig. 6a, b). Moreover, ARNILA knockdown failed to suppress the EMT programme when Sox 4 was ectopically expressed in MDAMB-231 cells, while ARNILA overexpression could not restore the EMT process when Sox 4 was knocked down in Hs578T cells with regard to the alteration of EMT markers (Fig. 6c). In contrast to ARNILA, miR-204 has a suppressive effect on migration and invasion, as anti-miR-204 significantly enhanced migration and invasion (Supplementary Figure S4). Moreover, decreased miR-204 restored the

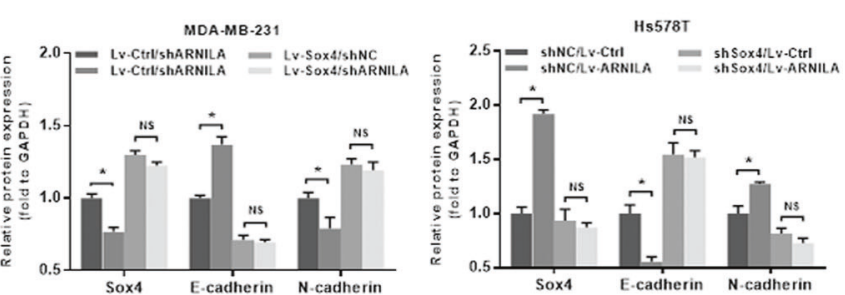

panel. c Left: western blotting assays of expression of E-cadherin and $\mathrm{N}$-cadherin in MDA-MB-231 and Hs578T cells following the indicated treatments. Right: quantification of protein levels shown in the left panels. The results are presented as the mean \pm SD. $* P<0.05$. NS indicates no significant difference

ARNILA knockdown-mediated repression of migration and invasion (Supplementary Figure S4). Taken together, our findings indicate that Sox4 is responsible for ARNILAmediated migration, invasion and EMT in TNBC cells.

\section{ARNILA promotes breast cancer metastasis in vivo}

In addition, we investigated the function of ARNILA in metastasis of breast cancer in vivo by injection of highly aggressive MDA-MB-231 cells that harboured shARNILA or shNC into the tail vein. As shown in Fig. 7a, PET/CT imaging revealed that injection of cells harbouring shNC resulted in lung and liver metastasis in BALB/c mice, whereas lung and liver metastases were significantly reduced in the mice injected with cells harbouring shARNILA. Histological examination confirmed the significantly reduced lung and liver metastases in the shARNILA group compared to the control group (lung, $P=0.013$; liver, $P=0.050$ ) (Fig. 7b, c). These data suggest that ARNILA contributes to TNBC metastasis and that silencing of ARNILA inhibits lung and liver metastases in mouse transplant models.

\section{Discussion}

The clinical management of TNBC is challenging because of its relatively aggressive biological behaviour and the lack 


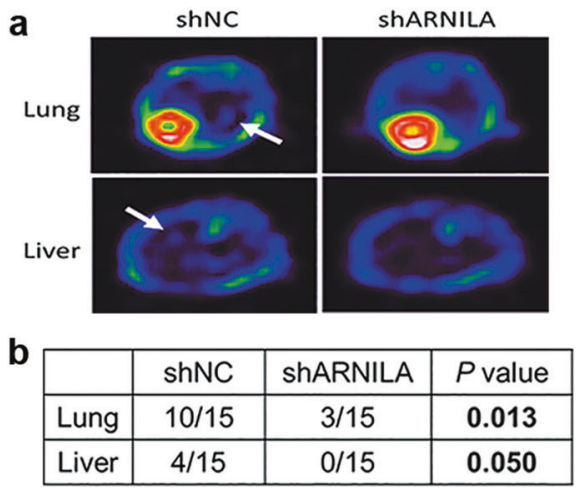

d

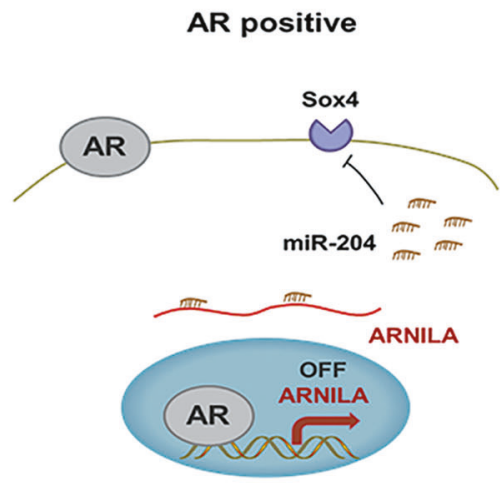

Fig. 7 ARNILA promotes metastasis in vivo. a Representative PET/ $\mathrm{CT}$ images of mice at $1 \mathrm{~h}$ after ${ }^{18} \mathrm{~F}-\mathrm{FDG}$ injection. Images were collected 5 weeks after cell injection. Lung and liver metastases are indicated by white arrows. b Representative images of metastatic nodules in lungs and livers by isolated lung bright-field imaging (top) and haematoxylin and eosin staining (middle and bottom). Lung and liver metastases are indicated by black arrows. $\mathbf{c}$ The incidence of lung and liver metastasis in two groups (Fisher's exact $t$ test). d A schematic

of effective therapeutic targets. AR has been reported to be expressed in a subset of TNBC patients and implicated in predicting clinical outcome. Emerging evidence has demonstrated that the AR signalling pathway functions in cancer processes. However, the underlying mechanisms by which AR functions in tumour progression as well as in predicting prognosis in TNBC remain unclear. In this study, we identified the IncRNA ARNILA, which was transcriptionally repressed by $\mathrm{AR}$, and showed that it was a promising therapeutic target that was functionally required for invasion and metastasis of TNBC. ARNILA promoted EMT by competitively binding to miR-204, leading to the upregulation of Sox4. In AR-positive tumours, ARNILA was apparently suppressed by AR, resulting in the decreased adsorption of miR-204, thereby releasing miR204 to inhibit Sox4 expression. Reciprocally, in ARnegative breast cancer, activated ARNILA promoted Sox4 expression by increasing the sequestration of miR-204 (Fig. 7d). It has been reported that Sox 4 is not only required but also sufficient for inducing EMT and metastatic spread

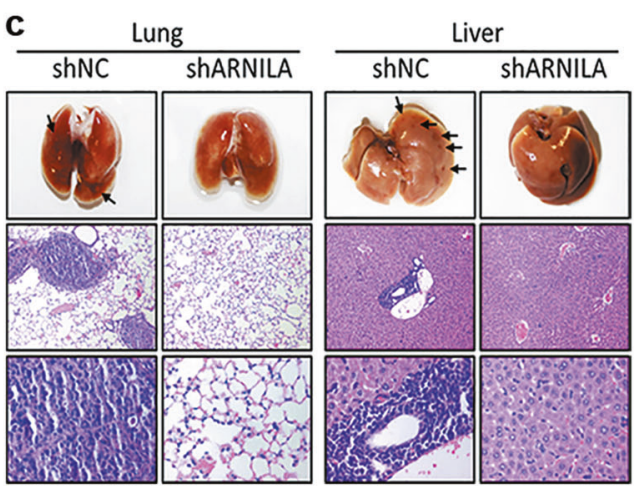

AR negative

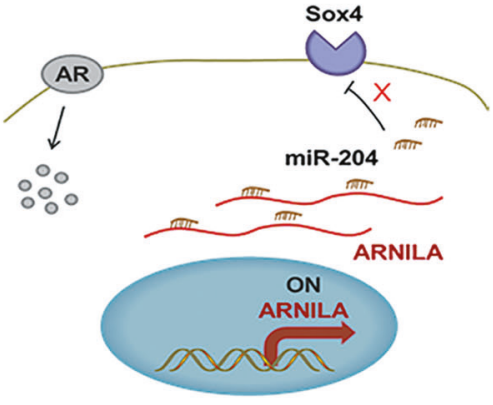

diagram of an ARNILA-based signalling circuit in TNBC. In ARpositive TNBC (left), AR significantly represses ARNILA by acting as a transcriptional repressor; hence, ARNILA's sponge-like function is weak here because of its low expression level, and miR-204 is released to inhibit Sox4. In AR-negative TNBC (right), miR-204 is mostly consumed by ARNILA due to the reduced repression by AR, and Sox 4 is consequently expressed at high levels

in breast cancer [18]. Therefore, ARNILA was demonstrated to function in metastasis of breast cancer.

In recent years, the roles of lncRNA in human diseases such as cancer have attracted increased attention. In breast cancer, several lncRNAs have been shown to have prognostic value or even be therapeutic options for patients. Reduced expression of NKILA, an lncRNA induced by nuclear factor $\mathrm{kB}(\mathrm{NFkB})$, in response to inflammatory signalling, was linked to metastatic dissemination and poor prognosis in breast cancer [21], suggesting that NKILA serves as a tumour suppressor. In contrast, overexpression of the lncRNA HOTAIR was highly predictive of poor metastasis-free survival or OS in early-stage surgically resected breast cancer. Enforced expression of HOTAIR increased the tumour invasiveness and metastasis of breast cancer cell lines [11]. Knockdown of MALAT1 led to slower tumour growth and reduced migration and metastasis in mouse mammary carcinoma model [35], indicating that HOTAIR and MALAT1 are potential therapeutic targets for breast cancer. Herein, we identified an uncharacterised 
IncRNA, ARNILA, that was correlated with decreased PFS in TNBC patients. Silencing ARNILA effectively inhibited cancer migration, invasion and metastasis, whereas enforced ARNILA expression promoted tumour progression. Therefore, ARNILA is probably another reliable prognostic biomarker and therapeutic target of TNBC patients.

Several mechanisms illustrating the regulation of cancer cellular process by IncRNA have been proposed, and the cellular location of IncRNA is important to its biological function. Generally, cytoplasmic lncRNAs influence cellular signalling cascades and modulate mRNA stability or translation, whereas nuclear lncRNAs are responsible for RNA processing, transcriptional regulation and chromatin interactions [36]. Increasing evidence has shown that cytoplasmic lncRNAs can act as "RNA sponges" or ceRNAs to sequester miRNAs and thereby reduce their regulatory effect on target mRNAs [37]. For example, lncARSA promoted sunitinib resistance by acting as a ceRNA via competitively binding miR-34/miR-449 to regulate AXL and c-MET in RCC cells [15]. Additionally, HOTAIR could sponge miR-331-3p to facilitate HER2 expression in gastric cancer by functioning as a ceRNA [38]. In our study, we identified the subcellular localisation of ARNILA and found that ARNILA predominately resided in the cytoplasm; thus, ARNILA might function as a ceRNA in TNBC. Subsequent bioinformatics analysis revealed an miRNA response element (MRE) of miR-204 shared by ARNILA and Sox4, and luciferase assays demonstrated that ARNILA and Sox4 both contained functional miR-204 binding sites. A positive regulatory relationship between ARNILA and Sox4 was observed by western blot analyses. Moreover, ARNILA was demonstrated to alter the expression of other miR-204 targets, including BCL2, RAB22A, SIRT1, and FOXA1. These results suggested the molecular sponge-like effect of ARNILA in facilitating expression of Sox4. However, appropriate transcript copy numbers for miRNA and miRNA target site abundance might be required to exert the ceRNA function [39]. We performed the absolute qRT-PCR analysis to quantify the copy numbers of ARNILA and miR-204 in TNBC cells overexpressed ARNILA or the control. Comparable copy numbers of ARNILA and miR204 were detected per cell in the control group, which is crucial for ceRNA-miRNA interaction. ARNILA overexpression significantly increased the copy numbers of ARNILA and decreased the copy numbers of miR-204 (Supplementary Figure S5), which probably led the miRNA target derepression at the high threshold of added MREs [39]. Further quantitative analyses of ARNILA, miR-204 and transcriptome-wide miR-204 targets might be needed to further verify the ceRNA hypothesis. Notably, in addition to the cytoplasm, ARNILA could also be located in the nucleus of TNBC cells, indicating other possible regulatory mechanisms of ARNILA in cellular biological processes, which require further validation in future work.

In conclusion, our findings first showed that AR negatively regulated the IncRNA ARNILA and provided a comprehensive annotation of this lncRNA in TNBC. We demonstrated that ARNILA was correlated with poor clinical outcome by modulating migration, invasion, EMT and metastasis. Therefore, ARNILA can act not only as a prognostic biomarker but also as a therapeutic target for TNBC patients.

\section{Materials and methods}

\section{Tissue samples}

Tissue samples were collected from 88 TNBC patients in The First People's Hospital of Lianyungang and The First Affiliated Hospital of Wenzhou Medical University, China. Inclusion criteria were female, age of 18 years or older, no distant metastasis at the time of diagnosis, and no neoadjuvant chemotherapy.

\section{Cell lines and culture}

MDA-MB-231 and Hs578T cells were cultured in RPMI 1640 and DMEM medium (Gibco, USA), respectively, supplemented with $10 \%$ fetal bovine serum (FBS, Gibco) and $1 \%$ penicillin/streptomycin in a $37^{\circ} \mathrm{C}$ incubator with $5 \% \mathrm{CO}_{2}$. MDA-MB-436 cells were cultured in L-15 medium (Gibco) supplemented with $10 \%$ FBS (Gibco) and $1 \%$ penicillin/streptomycin in a $37{ }^{\circ} \mathrm{C}$ incubator without $\mathrm{CO}_{2}$. DHT (Sigma, USA) was dissolved in ethanol, and cells were treated with DHT at $100 \mathrm{nM}$ for $24 \mathrm{~h}$. The same concentration of ethanol was used as the negative control treatment in parallel to DHT treatment.

\section{Plasmids and cell transfection}

Plasmids were transfected into cells by Lipofectamine 2000 (Invitrogen, USA) according to the instructions. The plasmids pGpU6/GFP/Neo-ARNILA, pGpU6/GFP/Neo-Sox4, pEX-2 (pGCMV/MCS/IRES/EGFP/Neo)-ARNILA, pEX-2-Sox4, LV5 (LV5-EF1a-GFP/Puro)-Sox4，LV5 (LV5-EF1a-GFP/ Puro)-ARNILA and LV3 (pGLV-h1-GFP-Puro)-ARNILA were purchased from GenePharma (Shanghai, China). Sequences were as follows: shARNILA1: 5'-GGTGCAGT TGCTATGGCAATG-3', shARNILA2: 5'- GAAGAGGCA TTCGGTGGATTC-3', shARNILA3: 5'-GGAGTCAGCTG ATGGGTTACA-3', shARNILA4: 5'-GCAATCGCACAAA GCAATTGT-3'; shSox4: 5'-TGGGCACATCAAGCGACC 
CAT-3'. The plasmid shARNILA3 was chosen for further study based on the silencing effect.

\section{RNA extraction, reverse transcription, and real-time RT-PCR}

Total RNA was extracted by TRIzol reagent (Invitrogen) and reverse-transcribed with a PrimeScript ${ }^{\mathrm{TM}} \mathrm{RT}$ reagent Kit with gDNA Eraser (Perfect Real Time) (TaKaRa, Japan). Primers were synthesised by Realgene (Nanjing, China) and include the following: forward ARNILA: 5'-AGGAGGCTGCAGGATGTA-3', reverse ARNILA: 5'-TATGGTTGGAGTC TTCTCCC-3'; forward GAPDH 5'-AGAAGGCTGGG GCTCATTTG-3', reverse GAPDH 5'-AGGGGCCATCCACAGTCTTC-3'; forward miR-204-5p: 5'-TTCCCTTTGT CATCCTATGCCT-3', reverse miR-204-5p: 5'- CCGACC GTTATATCGACATCTC-3'; forward U1: 5'-GGGAGATACCATGATCACGAAGGT-3', reverse U1: 5'-CCACAAATTATGCAGTCGAGTTTCCC-3'. Real-time PCR reactions were conducted with SYBR Premix Ex Taq ${ }^{\mathrm{TM}}$ II (TaKaRa). GAPDH was used as the endogenous control.

\section{Microarray analysis}

LncRNA expression profiling was performed by an Arraystar Human LncRNA Microarray V3.0 platform (Agilent Technologies). Differentially expressed lncRNAs were identified by $P$ value/false discovery rate filtering.

\section{ChIP assay}

ChIP assays were performed by a Pierce Agarose ChIP Kit (Thermo). Sequences of primers 1-10 used for ChIP are listed as follows. Forward $\left(5^{\prime}-3^{\prime}\right)$ : CCACAGTTAGCACAGGGAC, TCCCATTTTTTTTCCCCAATGGAAG, TG CACGGGCTGACTCCACAAA, GGCCATGTGCTTGCC GAC, ACCACTCCAATCACACACAC, GTTTTCTTAA AGAAAATCTTAAAACAAAC, CCCACCACAAGGAG CCCTCGAATTG, ACCCTTTGCGCAGAAGGCAACG, GACAGCTCAGTGGCTGGG, GAGTAGAAAATAAAA GGTCTGACCG; reverse (5'-3'): CAGTGAACCTCACCTATTGAATAAACC, TTACCTCTGTTGCCCTGATTGC, CCAAGTCCACGTCCCTAGAAAG, GTGTATTTCTTTT CTAGTACTTGGTCTG, CAAAAACCCAACACCCTTC CC, ATCAGAACTAATCCAGAGTG, TTCACCAAGGA TGGAGAAAGGGAGACAGC, GTGCCAGCACCATTC CTGGTC, TTTGGGTTTTGTTTCTTGCTGTGACTG, AC ATGGGGGCAAACTGAGG.

\section{RACE assay}

RACE was conducted by using a SMARTer RACE cDNA Amplification Kit (Clontech, Palo Alto, CA). The primers used for the nested-PCR are listed as follows: $5^{\prime}$ RACEouter: 5'-CGTGTCCGGCATGTAACCCATCAG-3', 5' RACE-inner: 5'-GGGAATCCACCGAATGCCTCTTCA3'; 3' RACE-outer: 5'-AGATGTTGTCTCTGCAGCACAAGGTAGG-3', 3' RACE-inner: 5'-GGTGCAGTTGCTATGGCAATGCCTACAG-3'.

\section{Immunohistochemistry (IHC) and FISH}

Tissue sections were deparaffinized and rehydrated, followed by antigen retrieval. For IHC, after primary anti-AR (ab198394, Abcam), anti-Sox4 (ab80261, Abcam) and secondary antibody incubation, the sections were incubated with diaminobenzidine (Sigma) and counterstained with haematoxylin (Sigma). For FISH, the probes of AR and ARNILA were synthesised by GenePharma (Shanghai, China).

\section{Luciferase reporter assay}

The wild-type or mutated sequences within the predicted binding sites of the 3'UTR sequence of ARNILA and Sox 4 were synthesised, inserted into a luciferase reporter plasmid (GENE, Shanghai, China), and transfected into 293T cells. The luciferase activity was normalised to Renilla luciferase activity after $48 \mathrm{~h}$ of transfection.

\section{Western blot}

The primary antibodies used include anti-AR (ab108341, Abcam), anti-Sox4 (ab80261, Abcam), anti-E-cadherin (ab40772, Abcam), anti-N-cadherin (ab76011, Abcam), anti-BCL2 (ab32124, Abcam), anti-FOXA1 (ab170933, Abcam), anti-RAB22A (ab137093, Abcam), anti-SIRT1 (ab32441, Abcam), anti-RUNX2 (ab23981, Abcam) and anti-GAPDH (10494-1-AP, Proteintech, USA) antibodies. The secondary antibody was anti-rabbit IgG-HRP (ab6721, Abcam).

\section{Migration and invasion assays}

Cells were seeded in six-well plates and grown to $90 \%$ confluence, and then, a wound was scratched in the cell monolayer with a $200 \mu \mathrm{L}$ sterile pipette tip. Cells were cultured in serum-free medium after removing floating cells with PBS. We photographed the edge of a scratch at time 0 and $24 \mathrm{~h}$. Transwell chambers (Corning, USA) were coated with $10 \mu \mathrm{L}$ of RPMI 1640 or DMEM-diluted Matrigel (BD Biosciences, USA), and cancer cells $\left(2 \times 10^{4}\right)$ cultured in serum-free medium $(200 \mu \mathrm{L})$ were added to the upper chamber. The medium $(800 \mu \mathrm{L})$ containing $10 \%$ FBS was added to the lower chamber. The cells in the upper side of the chamber were carefully removed with a cotton swab 
after $24 \mathrm{~h}$ incubation. The migrating cells were fixed with $100 \%$ methanol for $30 \mathrm{~min}$ and then stained with crystal violet for $10 \mathrm{~min}$.

\section{Tumour xenografts}

Animal studies were performed with approval of the Jinling Hospital Animal Care and Use Committee. Female BALB/c nude mice aged 4 weeks were injected with MDA-MB-231 cells $\left(1 \times 10^{7}\right)$ with stable knockdown of control or ARNILA vectors via tail veins. ${ }^{18} \mathrm{~F}-\mathrm{FDG}$ PET/CT was performed at 8 weeks after tumour cell injection to monitor the lung and liver metastasis. After PET/CT imaging, all mice were sacrificed to harvest the lung and liver tissues for haematoxylin and eosin staining.

\section{Statistical analysis}

Statistical analysis was performed by SPSS (v21), and the figures were plotted by GraphPad Prism software (v6). Student $t$ test and Fisher's exact $t$ test were used to examine statistical significance of differences between groups. $P$ values less than 0.05 were considered statistically significant, and all analyses were two sided. Data are presented as the mean $\pm \mathrm{SD}$.

Acknowledgements This work was supported by the National Natural Science Foundation of China (No. 81773102, No. 81470357) and a Foundation for Clinical Medicine Science and Technology Special Project of the Jiangsu Province, China (No. BL2014071) (to XG).

\section{Compliance with ethical standards}

Conflict of interest The authors declare that they have no conflict of interest.

\section{References}

1. Cancer Genome Atlas N. Comprehensive molecular portraits of human breast tumours. Nature. 2012;490:61-70.

2. Foulkes WD, Smith IE, Reis-Filho JS. Triple-negative breast cancer. N Engl J Med. 2010;363:1938-48.

3. Robson M, Im SA, Senkus E, Xu B, Domchek SM, Masuda N, et al. Olaparib for metastatic breast cancer in patients with a germline BRCA mutation. N Engl J Med. 2017;377:523-33.

4. Chang C, Lee SO, Yeh S, Chang TM. Androgen receptor (AR) differential roles in hormone-related tumors including prostate, bladder, kidney, lung, breast and liver. Oncogene. 2014;33: 3225-34.

5. Ricciardi GR, Adamo B, Ieni A, Licata L, Cardia R, Ferraro G, et al. Androgen receptor (AR), E-cadherin, and Ki-67 as emerging targets and novel prognostic markers in triple-negative breast cancer (TNBC) patients. PLoS ONE. 2015;10:e0128368.

6. Thike AA, Yong-Zheng Chong L, Cheok PY, Li HH, WaiCheong Yip G, Huat Bay B, et al. Loss of androgen receptor expression predicts early recurrence in triple-negative and basallike breast cancer. Mod Pathol. 2014;27:352-60.
7. Tang D, Xu S, Zhang Q, Zhao W. The expression and clinical significance of the androgen receptor and E-cadherin in triplenegative breast cancer. Med Oncol. 2012;29:526-33.

8. Mercer TR, Dinger ME, Mattick JS. Long non-coding RNAs: insights into functions. Nat Rev Genet. 2009;10:155-9.

9. Prensner JR, Iyer MK, Sahu A, Asangani IA, Cao Q, Patel L, et al. The long noncoding RNA SChLAP1 promotes aggressive prostate cancer and antagonizes the SWI/SNF complex. Nat Genet. 2013;45:1392-8.

10. Huarte M, Guttman M, Feldser D, Garber M, Koziol MJ, Kenzelmann-Broz D, et al. A large intergenic noncoding RNA induced by $\mathrm{p} 53$ mediates global gene repression in the p53 response. Cell. 2010;142:409-19.

11. Gupta RA, Shah N, Wang KC, Kim J, Horlings HM, Wong DJ, et al. Long non-coding RNA HOTAIR reprograms chromatin state to promote cancer metastasis. Nature. 2010;464:1071-6.

12. Fan Y, Shen B, Tan M, Mu X, Qin Y, Zhang F, et al. Long noncoding RNA UCA1 increases chemoresistance of bladder cancer cells by regulating Wnt signaling. FEBS J. 2014;281:1750-8.

13. Wang KC, Chang HY. Molecular mechanisms of long noncoding RNAs. Mol Cell. 2011;43:904-14.

14. Tsai MC, Manor O, Wan Y, Mosammaparast N, Wang JK, Lan F, et al. Long noncoding RNA as modular scaffold of histone modification complexes. Science. 2010;329:689-93.

15. Qu L, Ding J, Chen C, Wu ZJ, Liu B, Gao Y, et al. Exosometransmitted lncARSR promotes sunitinib resistance in renal cancer by acting as a competing endogenous RNA. Cancer Cell. 2016;29:653-68.

16. Thiery JP, Acloque H, Huang RY, Nieto MA. Epithelial - mesenchymal transitions in development and disease. Cell. 2009;139:871-90.

17. Zhang J, Liang Q, Lei Y, Yao M, Li L, Gao X, et al. SOX4 induces epithelial-mesenchymal transition and contributes to breast cancer progression. Cancer Res. 2012;72:4597-608.

18. Tiwari N, Tiwari VK, Waldmeier L, Balwierz PJ, Arnold P, Pachkov M, et al. Sox4 is a master regulator of epithelialmesenchymal transition by controlling Ezh2 expression and epigenetic reprogramming. Cancer Cell. 2013;23:768-83.

19. Gyorffy B, Lanczky A, Eklund AC, Denkert C, Budczies J, Li Q, et al. An online survival analysis tool to rapidly assess the effect of 22,277 genes on breast cancer prognosis using microarray data of 1,809 patients. Breast Cancer Res Treat. 2010;123:725-31.

20. Wang Y, Yang F, Wang Y, Zhong F, Guan X. Prognostic role of androgen receptor expression in triple-negative breast cancer. $\mathrm{J}$ Clin Oncol. 2015;33:(suppl; abstr 1076).

21. Liu B, Sun L, Liu Q, Gong C, Yao Y, Lv X, et al. A cytoplasmic NF-kappaB interacting long noncoding RNA blocks IkappaB phosphorylation and suppresses breast cancer metastasis. Cancer Cell. 2015;27:370-81.

22. Li JT, Wang LF, Zhao YL, Yang T, Li W, Zhao J, et al. Nuclear factor of activated $\mathrm{T}$ cells 5 maintained by Hotair suppression of miR-568 upregulates $\mathrm{S} 100$ calcium binding protein $\mathrm{A} 4$ to promote breast cancer metastasis. Breast Cancer Res. 2014;16:454.

23. Hou P, Zhao Y, Li Z, Yao R, Ma M, Gao Y, et al. LincRNA-ROR induces epithelial-to-mesenchymal transition and contributes to breast cancer tumorigenesis and metastasis. Cell Death Dis. 2014;5:e1287.

24. Sas-Chen A, Aure MR, Leibovich L, Carvalho S, Enuka Y, Korner C, et al. LIMT is a novel metastasis inhibiting lncRNA suppressed by EGF and downregulated in aggressive breast cancer. EMBO Mol Med. 2016;8:1052-64.

25. Latorre E, Carelli S, Raimondi I, D’Agostino V, Castiglioni I, Zucal C, et al. The ribonucleic complex HuR-MALAT1 represses CD133 expression and suppresses epithelial-mesenchymal transition in breast cancer. Cancer Res. 2016;76:2626-36. 
26. Liu YN, Liu Y, Lee HJ, Hsu YH, Chen JH. Activated androgen receptor downregulates E-cadherin gene expression and promotes tumor metastasis. Mol Cell Biol. 2008;28: 7096-108.

27. Kozomara A, Griffiths-Jones S. miRBase: annotating high confidence microRNAs using deep sequencing data. Nucleic Acids Res. 2014;42(Database issue):D68-73.

28. John B, Enright AJ, Aravin A, Tuschl T, Sander C, Marks DS. Human microRNA targets. PLoS Biol. 2004;2:e363.

29. Lanczky A, Nagy A, Bottai G, Munkacsy G, Szabo A, Santarpia L, et al. miRpower: a web-tool to validate survival-associated miRNAs utilizing expression data from 2178 breast cancer patients. Breast Cancer Res Treat. 2016;160:439-46.

30. Parvani JG, Schiemann WP. Sox4, EMT programs, and the metastatic progression of breast cancers: mastering the masters of EMT. Breast Cancer Res. 2013;15:R72.

31. Ying Z, Li Y, Wu J, Zhu X, Yang Y, Tian H, et al. Loss of miR204 expression enhances glioma migration and stem cell-like phenotype. Cancer Res. 2013;73:990-9.

32. Zhou X, Li L, Su J, Zhang G. Decreased miR-204 in H. pyloriassociated gastric cancer promotes cancer cell proliferation and invasion by targeting SOX4. PLoS ONE. 2014;9:e101457.
33. Yin JJ, Liang B, Zhan XR. MicroRNA-204 inhibits cell proliferation in T-cell acute lymphoblastic leukemia by downregulating SOX4. Int J Clin Exp Pathol. 2015;8:9189-95.

34. Wu D, Pan H, Zhou Y, Zhang Z, Qu P, Zhou J, et al. Upregulation of microRNA-204 inhibits cell proliferation, migration and invasion in human renal cell carcinoma cells by downregulating SOX4. Mol Med Rep. 2015;12:7059-64.

35. Arun G, Diermeier S, Akerman M, Chang KC, Wilkinson JE, Hearn S, et al. Differentiation of mammary tumors and reduction in metastasis upon Malat1 lncRNA loss. Genes Dev. 2016;30: $34-51$.

36. Schmitt AM, Chang HY. Long noncoding RNAs in cancer pathways. Cancer Cell. 2016;29:452-63.

37. Tay Y, Rinn J, Pandolfi PP. The multilayered complexity of ceRNA crosstalk and competition. Nature. 2014;505:344-52.

38. Liu XH, Sun M, Nie FQ, Ge YB, Zhang EB, Yin DD, et al. Lnc RNA HOTAIR functions as a competing endogenous RNA to regulate HER 2 expression by sponging miR-331-3p in gastric cancer. Mol Cancer. 2014;13:92.

39. Denzler R, Agarwal V, Stefano J, Bartel DP, Stoffel M. Assessing the ceRNA hypothesis with quantitative measurements of miRNA and target abundance. Mol Cell. 2014;54:766-76. 